\title{
Factores de riesgo de homicidio de la mujer en la relación de pareja*
}

\section{Homicide Risk Factors Against Woman in a Couple Relationship}

Recibido: agosto 16 de 2012 | Revisado: mayo 26 de 2013 | Aceptado: octubre 3 de 2013

\section{LORENA CONTRERAS TAIBO **}

Universidad Diego Portales, Chile

doi:10.11144/Javeriana.UPSY13-2.frhm

Para citar este artículo: Contreras, L. (2014). Factores de riesgo de homicidio de la mujer en la relación de pareja. Universitas Psychologica, 13(2), 681-692. doi:10.11144/Javeriana.UPSY13-2.frhm

* Artículo de revisión

*** Directora Clínica Psicológica, Facultad de Psicología, Universidad Diego Portales, Santiago de Chile. Presidenta Fundación Instituto Chileno para el Estudio de la Violencia, Santiago de Chile. Correo electrónico: lorena.contreras@udp.cl

\section{RESUMEN}

Con base en una revisión sistemática de estudios empíricos publicados entre los años 2000 y 2011, se identificaron los factores de riesgo de homicidio en contra de la mujer en el contexto de las relaciones de pareja, a partir de 17 investigaciones realizadas en EE. UU, Australia, España, el Reino Unido y Países Bajos. Particularmente, se analizan y discuten los hallazgos respecto a los factores de riesgo desde la perspectiva del perpetrador, la víctima, su relación de pareja y su contexto. Finalmente, se concluye la necesidad de determinar factores específicos de riesgo para la realidad latinoamericana, problematizando también en torno a las repercusiones de las políticas públicas en materia de violencia contra la mujer y su impacto en el nivel de riesgo vital experimentado por ellas.

Palabras clave

Femicidio, uxoricidio, homicidio, violencia doméstica, riesgo.

\section{A B S T R ACT}

Based on a systematic review of empirical studies published between 2000 and 2011, it is identified homicide risk factors against women in the context of couple relationships, from 17 researches in USA, Australia, Spain, the UK and Netherlands. In particular, it is analyzes and discusses findings regarding to the risk factors from the focus of the perpetrator, the victim, the couple relationship and its context. Finally, it is concludes the need to identify and determinate specific risk factors for the Latin American context, discussing about the impact of public policies on violence against women and its influence on the level of life risk they experience.

Keywords

Femicide, uxoricide, homicide, domestic violence, risk. 


\section{Introducción}

El fenómeno de la violencia doméstica constituye una práctica generalizada y profundamente arraigada (Organización Mundial de la Salud [OMS], 2005), con una elevada prevalencia a nivel mundial, siendo una de las formas más recurrentes aquella ejercida contra la mujer por parte de su pareja o expareja (Krug, Dahlberg \& Mercy, 2003). Al respecto, Heise, Ellsberg y Gottemoeller (1999) analizaron 50 encuestas poblacionales a nivel mundial, hallando que entre el 10\% y el 50\% de las mujeres reconocen haber padecido abuso físico por parte de su pareja alguna vez en su vida. Este maltrato, de acuerdo a la dinámica victimal propia de este fenómeno, se asocia a agresiones reiteradas en el tiempo, así como a manifestaciones polimórficas, incluyendo maltrato físico, abuso psicológico, violencia sexual y violencia económica.

Esta violencia hacia la mujer en el contexto de pareja no solo acarrea severas consecuencias a nivel personal y relacional, sino que puede llegar a costar la vida de la víctima, fenómeno denominado como "femicidio". Este concepto ha sido definido por Naciones Unidas (Programa de las Naciones Unidas para el Desarrollo [PNUD], 2004) como:

El asesinato de mujeres como resultado extremo de la violencia de género, que ocurre tanto en el ámbito privado como público y, comprende aquellas muertes de mujeres a manos de sus parejas, ex parejas (sic) o familiares, asesinadas por acosadores, agresores sexuales y/o violadores, así como aquellas que trataron de evitar la muerte de otra mujer y quedaron atrapadas en la acción femicida.

Dicha noción ha sido traducida también como "feminicidio", concepto que pretende hacerse cargo de la mirada de género desde la que se sustenta, siendo definido como el "asesinato de mujeres por hombres por ser mujeres" (Russell \& Harmes, 2006, p. 76).

Estadísticas de diferentes países indican que el homicidio de la mujer en el contexto de pareja constituye un grave problema mundial, revelando que "entre el 40\% y 70\% de los asesinatos de mu- jeres las víctimas fueron muertas por su esposo o novio, a menudo en el contexto de una relación de maltrato constante" (Krug et al., 2003, p. 101). De este modo, estudios recientes señalan que la media internacional de femicidios íntimos es de 5.04 por millón de mujeres, incrementándose a un 8.59 en el caso de América Latina (Sanmartín, Iborra, García \& Martínez, 2010).

Este fenómeno, estudiado desde principios de la década del 90, se hace evidente en América Latina a partir de los múltiples hechos ocurridos en México y, particularmente, en Ciudad Juárez (Instituto Interamericano de Derechos Humanos [IIDH] $\&$ Consejo Centroamericano de Procuradores de Derechos Humanos [CCPDH], 2006), erigiéndose como un problema de seguridad ciudadana. Respecto al particular, expertos en los campos de la psicología, criminología y victimología han sido enfrentados a una demanda social vinculada a la valoración o predicción del riesgo de conductas violentas, solicitud que tiene por objeto gestionar el riesgo identificado, previniendo de este modo la futura ocurrencia de hechos de sangre en el ámbito doméstico.

Por tanto, el presente artículo pretende realizar un recorrido, con miras a identificar aquellos aportes empíricos referidos a la evaluación de riesgo de violencia letal hacia la mujer en la relación de pareja, para centrarse particularmente en los factores de riesgo que permiten predecir el resultado de asesinato en la mujer.

\section{Método}

Entre los meses de febrero y junio de 2012, se realizó un análisis sistemático de la literatura, con el objetivo de contestar la siguiente pregunta de investigación: de acuerdo a la bibliografía especializada a nivel internacional, icuáles son los factores de riesgo que empíricamente han demostrado relación con el asesinato de la mujer en el contexto de una relación de pareja? Para responder esta pregunta, se consultaron las bases EBSCO, Jstore, Sage y Psicodoc; utilizando las siguientes palabras clave en español: femicidio, uxoricidio, homicidio, violencia doméstica y riesgo, y en inglés: femicide, uxoricide, 
homicide, domestic violence, risk. Con el fin de obtener resultados de investigaciones actualizadas, se acotó la búsqueda al período comprendido entre los años 2000 y 2011.

Dado que la aspiración futura de esta revisión documental dice relación con poder aplicar sus hallazgos a la realidad latinoamericana, se seleccionaron aquellos estudios realizados en culturas occidentales, las cuales resultan relativamente comparables con nuestra realidad. Por esta razón, en relación con la carga cultural del tema examinado, se excluyeron aquellos estudios desarrollados en los continentes de África y Asia, en los cuales se presumen patrones culturales que distan mucho de los latinoamericanos. Del mismo modo, se excluyeron aquellos estudios cuyo nivel de representatividad estuviera circunscrito a poblaciones locales muy específicas, tal como alguna ciudad particular dentro de un Estado. Esto, por cuanto la extrapolación de sus resultados a un contexto como el nuestro, resulta en extremo dudosa y riesgosa.

De esta forma, fueron incluidas todas las investigaciones de carácter empírico, realizadas en poblaciones occidentales, en las cuales se estudiaron factores que potencialmente se refirieran a la probabilidad de asesinato a la mujer, en el contexto de una relación de pareja heterosexual, población mayoritaria en este tipo de delitos.

Con base en dichos criterios de inclusión y exclusión, se revisaron los títulos de los artículos, a fin de cotejar su pertinencia en relación al tema abordado. Luego de este primer filtro, se revisaron los resúmenes de las investigaciones, con el objetivo de identificar aquellos artículos que se referían específicamente a investigaciones empíricas en factores de riesgo de letalidad en relaciones íntimas. Producto de esta revisión discriminante, se seleccionaron un total de 37 artículos, de los cuales se realizó una lectura completa, con el objeto de determinar su pertinencia a los objetivos de esta investigación. Finalmente, con base en el procedimiento anteriormente señalado, se incluyeron 17 investigaciones empíricas que guardan relación con factores de riesgo de asesinato de la mujer en el contexto de relación de pareja.

\section{Resultados}

Cabe señalar que las distintas investigaciones estudiadas manejan criterios de inclusión de la muestra diversos, en virtud del tipo de relación considerada entre sus miembros, tales como uxoricidio, relaciones de pareja con convivencia o sin ella, entre otros. Por otra parte, los estudios difieren respecto a la naturaleza y severidad de la violencia estudiada, contemplando homicidio frustrado, consumado, y/o violencia grave. Asimismo, difieren respecto a los grupos que comparan, a saber: homicidas de mujeres vs. homicidas de hombres, homicidas de pareja con y sin suicidio, víctimas de violencia letal y no letal, entre otros. Por otra parte, los estudios emplean diferentes unidades de análisis, pudiendo obtener información a partir de víctimas, imputados, informes, estadísticas, entre otros. Todo ello lleva a resultados y conclusiones diferenciales, dependiendo del foco del investigador.

Junto con esto, muchos de estos estudios no plantean el objetivo de levantar propiamente factores de riesgo para el homicidio de la mujer en contexto de pareja, razón por la cual ha sido necesario extrapolar de sus hallazgos aquellas variables que pueden ser consideradas como factores de riesgo, en tanto se asocian con la probabilidad de desencadenar este resultado.

A continuación, en la Tabla 1 se detallan los 17 estudios utilizados para el análisis, dando cuenta de sus autores y año de publicación, la muestra que emplearon y el país en el cual fueron desarrollados (EE. UU., Reino Unido, España, Australia y Países Bajos), así como los factores de riesgo que abordan.

En esta tabla, se puede apreciar que se han desarrollado fundamentalmente estudios censales, con base en el análisis de estadísticas, y estudios muestrales, cuyo diseño no siempre es controlado.

A continuación, se procederá a detallar los factores de riesgo de asesinato hacia la mujer en la relación de pareja, identificados por los estudios en la materia. Para organizar esta información, los factores de riesgo han sido agrupados de acuerdo al agente, es decir, variables propias del autor, de la víctima, de la relación o del contexto. 


\section{TABLA 1}

Estudios considerados respecto a factores de riesgo de asesinato a la mujer en la pareja

\begin{tabular}{|c|c|c|}
\hline Referencia & Muestra & Factores de riesgo estudiados \\
\hline $\begin{array}{l}\text { Puzone, Saltzman, Kres- } \\
\text { now, Thompson y Mercy } \\
(2000) \text {. }\end{array}$ & $\begin{array}{l}\text { Estadísticas de } 44.893 \text { homicidios ín- } \\
\text { timos entre } 1976 \text { y } 1995 \text { (EE. UU ). }\end{array}$ & Estado civil, raza. \\
\hline $\begin{array}{l}\text { Sharps, Campbell, Cam- } \\
\text { pbell, Gary y Webster } \\
\text { (2001). }\end{array}$ & $\begin{array}{l}380 \text { víctimas de homicidio con- } \\
\text { sumado y frustrado, } 384 \text { mujeres } \\
\text { maltratadas y } 376 \text { no maltratadas } \\
\text { (EE. UU.). }\end{array}$ & Consumo problemático de alcohol. \\
\hline $\begin{array}{l}\text { McFarlane, Campbell y } \\
\text { Watson (2002). }\end{array}$ & $\begin{array}{l}384 \text { víctimas de violencia doméstica } \\
\text { y } 437 \text { víctimas de homicidio íntimo, } \\
\text { frustrado o consumado (EE. UU.). }\end{array}$ & Acoso y amenazas. \\
\hline Campbell et al. (2003). & $\begin{array}{l}220 \text { casos de homicidio y } 343 \text { casos } \\
\text { de maltrato domestico (EE. UU.). }\end{array}$ & $\begin{array}{l}\text { Escalada de violencia física, acceso a armas, separación, desempleo, utiliza- } \\
\text { ción o amenaza con armas, intentos de homicidio, evasión de detención por } \\
\text { VIF, hijo no biológico del agresor, sexo forzado, intento de estrangulamien- } \\
\text { to, uso de drogas, problemas con el alcohol, conducta controladora, celos, } \\
\text { maltrato durante el embarazo, intento de suicidio, amenazas de daño a los } \\
\text { niños, percepción de riesgo vital, acoso. }\end{array}$ \\
\hline $\begin{array}{l}\text { Dugan, Rosenfield y Na- } \\
\text { gin (2003). }\end{array}$ & $\begin{array}{l}\text { Estadísticas de homicidio en la pare- } \\
\text { ja y recursos para violencia domés- } \\
\text { tica, en } 48 \text { ciudades entre } 1.976 \text { y } \\
1.996 \text { (EE. UU.). }\end{array}$ & Recursos disponibles para la prevención de violencia doméstica. \\
\hline $\begin{array}{l}\text { Decker, Martin y Morac- } \\
\text { co (2004). }\end{array}$ & $\begin{array}{l}53 \text { mujeres embarazadas, maltrata- } \\
\text { das por su pareja durante el embara- } \\
\text { zo (EE. UU.). }\end{array}$ & Embarazo. \\
\hline $\begin{array}{l}\text { Dobash, Dobash, Cava- } \\
\text { nagh y Lewis (2004). }\end{array}$ & $\begin{array}{l}106 \text { homicidas de pareja femenina y } \\
424 \text { homicidas de hombres (Reino } \\
\text { Unido). }\end{array}$ & $\begin{array}{l}\text { Violencia hacia parejas anteriores, consumo de alcohol, actividad criminal, } \\
\text { separación. }\end{array}$ \\
\hline McFarlane et al. (2005). & $\begin{array}{l}48 \text { mujeres víctima de violencia } \\
\text { física y } 100 \text { de violencia sexual (EE. } \\
\text { UU.). }\end{array}$ & Agresión sexual. \\
\hline $\begin{array}{l}\text { Shackelford y Mouzos } \\
\text { (2005). }\end{array}$ & $\begin{array}{l}\text { Estadísticas de } 15.670 \text { homicidios } \\
\text { íntimos en EEUU y } 456 \text { en Austra- } \\
\text { lia (EE. UU. y Australia). }\end{array}$ & Estado civil, edad y diferencia de edad de los miembros de la pareja. \\
\hline Soria (2005). & $\begin{array}{l}46 \text { reos por violencia doméstica, } 20 \\
\text { homicidas y } 26 \text { maltratadores (Es- } \\
\text { paña). }\end{array}$ & Acoso. \\
\hline $\begin{array}{l}\text { Richardson y Mercy } \\
\text { (2006). }\end{array}$ & $\begin{array}{l}\text { Estadísticas de } 966 \text { homicidios en } \\
\text { la pareja, en estados que tienen o } \\
\text { no leyes que restringen el acceso a } \\
\text { armas (EE. UU.). }\end{array}$ & Leyes de restricción para la adquisición y tenencia de armas de fuego. \\
\hline $\begin{array}{l}\text { Dobash, Dobash, Ca- } \\
\text { vanagh y Medina-Ariza } \\
\text { (2007). }\end{array}$ & $\begin{array}{l}122 \text { hombres convictos por violencia } \\
\text { no letal a la pareja y } 106 \text { por violen- } \\
\text { cia letal (Reino Unido). }\end{array}$ & $\begin{array}{l}\text { Desempleo; posesividad y celos; separación; utilización de violencia con- } \\
\text { tra una pareja anterior; agresión sexual; estrangulamiento a la víctima; } \\
\text { utilización de un arma o instrumento. }\end{array}$ \\
\hline $\begin{array}{l}\text { Echeburúa y Fernández- } \\
\text { Montalvo (2007). }\end{array}$ & $\begin{array}{l}162 \text { agresores de violencia de pareja } \\
\text { (España). }\end{array}$ & Psicopatía. \\
\hline $\begin{array}{l}\text { Dixon, Hamilton-Gia- } \\
\text { chritsis y Browne (2008). }\end{array}$ & $\begin{array}{l}90 \text { sujetos encarcelados por el asesi- } \\
\text { nato de su pareja (Reino Unido). }\end{array}$ & Criminalidad y psicopatología. \\
\hline $\begin{array}{l}\text { Echeburúa, Fernández- } \\
\text { Montalvo, de Corral y } \\
\text { López-Goñi (2009). }\end{array}$ & $\begin{array}{l}269 \text { casos de violencia severa y } 812 \\
\text { de violencia menos severa (España). }\end{array}$ & $\begin{array}{l}\text { Inmigración, separación, acoso, violencia física, violencia física frente a } \\
\text { familiares, incremento de la violencia, amenazas graves, armas, lesiones, } \\
\text { agresión sexual, celos, violencia hacia parejas anteriores, violencia previa } \\
\text { hacia otros, alcohol/drogas, enfermedad mental, conducta cruel, justifica- } \\
\text { ción de la violencia, percepción de riesgo vital, retractación, vulnerabilidad } \\
\text { de la víctima. }\end{array}$ \\
\hline $\begin{array}{l}\text { Liem, Hengeveld, y Koen- } \\
\text { raadt (2009). }\end{array}$ & $\begin{array}{l}77 \text { homicidas con para-suicidio, } \\
430 \text { homicidas y } 161 \text { para-suicidas } \\
\text { (Países bajos). }\end{array}$ & $\begin{array}{l}\text { Temor al abandono, dependencia, ira narcisista, separación, desorden } \\
\text { depresivo del agresor. }\end{array}$ \\
\hline Dobash y Dobash (2011). & $\begin{array}{l}104 \text { sujetos encarcelados por el ho- } \\
\text { micidio de su pareja (Reino Unido). }\end{array}$ & $\begin{array}{l}\text { Negación de los hechos y/o su responsabilidad, historia de violencia previa } \\
\text { con la víctima y otras parejas, dificultades en las relaciones íntimas, con- } \\
\text { ducta de abuso y control, posesividad y celos, negación de responsabilidad, } \\
\text { culpabilización de la víctima, ausencia de remordimiento y empatía hacia la } \\
\text { víctima, resistencia al cambio. }\end{array}$ \\
\hline
\end{tabular}

Fuente: elaboración propia 


\section{Factores de riesgo asociados al perpetrador}

En relación con variables sociodemográficas, Echeburúa et al. (2009) han detectado una relación significativa entre el asesinato de la mujer por parte de su pareja y la inmigración, lo cual puede vincularse a situaciones de estrés, aislamiento y condiciones de precariedad. No obstante, Latinoamérica registra un comportamiento bastante disímil y heterogéneo respecto a fenómenos migratorios, con lo cual la comparación con la realidad europea resulta, cuando menos, arriesgada.

En la misma línea del estrés psicosocial, es posible interpretar la situación de desempleo del agresor, en tanto variable sociodemográfica más íntimamente relacionada con el fenómeno (Campbell et al., 2003), encontrándose la mayoría de los sujetos que ultimaron a su pareja sin ocupación al momento de los hechos (Dobash et al., 2007).

Por otra parte, existe una discusión en la literatura respecto al papel de las drogas y el alcohol, en el incremento del riesgo de violencia letal en la relación. Respecto a la utilización de sustancias, Campbell et al. (2003) consideran que el uso de drogas incrementa el riesgo de violencia letal, relación que no se ha podido establecer claramente con el consumo de alcohol. Esto concuerda con los hallazgos de Dobash et al. (2007), puesto que los homicidas conyugales resultan menos propensos a estar ebrios al momento del delito que los agresores de VIF. En este sentido, solo el 20\% se encontraban bebidos al momento del homicidio, mientras que el 7.9\% había consumido drogas ilegales (Dobash et al., 2004). Por el contrario, Sharps et al. (2001) concluyen que el consumo problemático de alcohol por parte del agresor duplica el riesgo de violencia letal en las relaciones de pareja, conclusión avalada también por Echeburúa et al. (2009).

Otra dimensión posible de vincular con el homicidio de pareja, son los antecedentes de violencia en la biografía del autor. A este respecto, diversos autores señalan la existencia de episodios de violencia hacia parejas anteriores (Dobash et al., 2004; Dobash et al., 2007; Echeburúa et al., 2009), registrando una mayor prevalencia en comparación a agresores domésticos (Dobash et al., 2007) y homicidas comunes (Dobash et al., 2004).
Junto con la violencia hacia parejas previas, también se han identificado manifestaciones de violencia hacia terceros (Echeburúa et al., 2009), lo cual se vincula con actividades criminales persistentes en un 59.6\% de los casos (Dobash et al., 2004). Estas manifestaciones de violencia pueden correlacionarse con la tenencia de armas, particularmente de fuego. En este sentido, el acceso a armas por parte del agresor se asocia estrechamente con el riesgo de homicidio. Por el contrario, el acceso a una única arma por parte de la víctima disminuye el riesgo (Campbell et al., 2003). Cabe señalar que esta realidad resulta escasamente comparable con lo que puede ocurrir en naciones latinoamericanas, en donde tienden a predominar otros medios comisivos, como las armas blancas.

Desde la perspectiva de salud mental, se discute la existencia de psicopatología por parte del agresor. Al respecto, Echeburúa et al. (2009) consideran que el historial de enfermedad mental, así como el abandono de terapias psicológicas y psiquiátricas, incrementan el riesgo de violencia grave en la relación de pareja. Del mismo modo, Dixon et al. (2008) han identificado que la presencia de psicopatología en conjunción con la criminalidad del autor, constituye un factor de riesgo en este tipo de delito. Al analizar los tipos de cuadro psicopatológico asociados, Echeburúa y Fernández-Montalvo (2007) descartan el papel de la psicopatía, al no encontrar una prevalencia significativa de dicho trastorno en la muestra estudiada; en tanto, los hallazgos de Liem et al. (2009) vinculan el uxoricidio con parasuicidio con la presencia de un desorden depresivo al momento de los hechos. Concordantemente con el señalamiento de sintomatología disfórica, las amenazas o intentos de suicidio por parte del agresor constituyen una variable predictora muy relevante (Campbell et al., 2003), pudiendo estar asociado con psicopatología y desesperanza, estados que no permiten valorar salidas alternativas a la finalización radical del conflicto. Por su parte, Liem et al. (2009, p. 509), entienden el ulterior suicidio del autor como "el resultado de sentimientos de vergüenza o culpa después del homicidio, así como temor a las consecuencias judiciales". 


\section{Factores de riesgo asociados a las víctimas}

A este respecto, probablemente la dimensión sociodemográfica sea una de las que generan más interrogantes respecto a su aplicabilidad al contexto latinoamericano, dado que resulta en extremo sensible a la realidad de cada país. Esto ocurre, por ejemplo, respecto a la raza de las víctimas, ámbito en el cual Puzone et al. (2000) identifican en EE. UU. un mayor riesgo total en las víctimas afroamericanas, en relación con las de raza blanca. Esto indica que este tipo de ilícitos tiende a afectar más frecuentemente a población en condiciones de vulnerabilidad social, o bien en situación de estrés psicosocial (Echeburúa et al., 2009). En esta misma línea, la letalidad ha sido asociada a la condición de vulnerabilidad de la víctima, debido a enfermedad, soledad o bien dependencia del agresor (Echeburúa et al., 2009), pues puede dificultar la interrupción de la relación e incluso la identificación de la vulneración asociada a la violencia ejercida en su contra.

Ello puede vincularse a la percepción subjetiva de riesgo vital en la víctima (Campbell et al., 2003; Echeburúa et al., 2009), relación no del todo lineal con el riesgo asociado. Ya sea por una suerte de minimización, por temor o por necesidad, las mujeres frecuentemente intentan retirar las denuncias previas por VIF, o bien echan pie atrás en la decisión de abandonar al agresor o denunciarlo. Para Echeburúa et al. (2009), la retractación por parte de la mujer constituye una variable predictora del espectro de violencia grave, en la cual se inscribe aquella de consecuencias fatales.

Junto con esto, la existencia de un hijo en el hogar sin lazo biológico con el perpetrador incrementa el riesgo de homicidio a la mujer en la pareja en más del doble (Campbell et al., 2003); puesto que este lazo puede agudizar los celos recurrentes e incluso violentos del agresor, en tanto recordatorio permanente de un vínculo que lo excluye.

\section{Factores de riesgo asociados a la relación}

Se han estudiado las características del vínculo que une a la víctima y al autor en el delito de homicidio en la pareja, arribando a hallazgos un tan- to disímiles. Por una parte, estudios comparados (Shackelford \& Mouzos, 2005) han determinado que el riesgo al que se expone la mujer es mucho mayor en una relación de convivencia, respecto a una relación marital (9.5 veces en Australia y 8.9 veces en EE. UU.). La razón de ello es que las parejas en situación de convivencia presentan niveles de separación superiores, lo cual se puede asociar a una sensación de control más precario sobre la relación y, por tanto, una necesidad creciente de mantener este control por la fuerza. Por el contrario, Puzone et al. (2000) concluyen que la mayor parte de las víctimas de homicidio en la pareja han sido ultimados por su cónyuge, constituyéndose por tanto el vínculo matrimonial en un factor de riesgo.

Respecto a la diferencia de edad entre la víctima y el agresor, las conclusiones de las investigaciones son dispares. Por una parte Dobash et al. (2007) han encontrado que las diferencias de edad de la víctima y el agresor no resultan estadísticamente significativas al momento de los hechos. No obstante, Shackelford y Mouzos (2005, p. 1321) concluyen que "el riesgo de uxoricidio se incrementa con la diferencia de edad en la pareja". Ahora bien, la discrepancia de resultados en distintas investigaciones puede tener que ver con las poblaciones estudiadas, resultando distinta esta diferencia etaria en parejas que mantienen o no vínculo civil.

Otro de los factores de riesgo más extensamente estudiados en la literatura es la convivencia y la interrupción de la relación, en tanto variable que incide en la probabilidad de homicidio hacia la mujer en la pareja (Echeburúa et al., 2009). Sobre el particular, se ha mencionado que no haber convivido nunca con el agresor constituye un factor protector de femicidio; por el contrario, la separación o la solicitud de que el agresor abandone el hogar común incrementa el riesgo asociado, particularmente durante el primer año (Campbell et al., 2003). Estos hallazgos no son compartidos por Dobash et al. (2007), quienes concluyen que las relaciones sin convivencia aumentan significativamente el riesgo letal, lo cual vinculan con un menor compromiso, mayor conflicto y menos apoyos externos, particularmente en un hombre posesivo. 
Sin embargo, y consistentemente con estudios anteriores, la separación o el rompimiento fue identificado como un factor de riesgo elevado, particularmente cuando este se asocia a celos exacerbados y posesividad del autor. Es este sentido, Dobash et al. (2004) señalan que "aproximadamente 1 de cada 3 mujeres estaban separadas de su pareja y aproximadamente 1 de cada 20 estaban tratando de terminar la relación. De importancia es el hecho de que era la mujer y no el hombre que parecía estar terminando o haber finalizado la relación, y así el distanciamiento de la mujer parecía ser la fuente del conflicto" (p. 597). De este modo, Liem et al. (2009), en los casos de uxoricidio, determinan que uno de los móviles principales para cometer el homicidio es el temor al abandono (casi el 50\% de los casos), lo cual refleja la dependencia del hombre, involucrado muchas veces en una relación con características simbiótica. En este caso, "el suicidio responde a un deseo de reunirse con la víctima en la muerte, manteniendo una relación que no ya es viable en vida" (p. 509). Otro de los motivos de uxoricidio sería la ira narcisista: "cuando la autoestima es baja y está amenazada por el rechazo, la agresión se presenta como un instrumento de recuperación del sentido de sí mismo" (p. 509).

Otras de las dimensiones sobre las cuales se han realizado numerosos estudios son las cualidades de la relación de violencia experimentada por la pareja. Al respecto, Dobash y Dobash (2011) señalan que los homicidas domésticos presentan un historial de violencia grave y repetida hacia la mujer que ultimaron. De hecho, Campbell et al. (2003) señalan que un $72 \%$ de las mujeres que murieron a manos de su pareja, habían sufrido previamente malos tratos. Sin embargo, esto quiere decir que el $28 \%$ de las víctimas no había vivido esta forma de violencia previa, cifra que para Dobash et al. (2007) asciende a 41\%, de acuerdo a las datos provenientes de los registros de sujetos condenados. Pese a la variabilidad de esta cifra, resulta evidente que hay un porcentaje de casos significativo donde no se produce una progresión o escalada de violencia, que permita anticipar el resultado letal. Además de ello, no todas las formas de violencia física preexistente se relacionan con un resultado fatal, siendo necesario contemplar aquella que se produce en presencia de familiares y la que se agudiza a lo largo del tiempo, así como aquella que genera lesiones intencionales en la víctima (Echeburúa et al., 2009).

Respecto al maltrato durante el embarazo, Campbell et al. (2003) identificaron que el 25.8\% de los asesinatos de mujeres por parte de sus parejas tenían antecedentes de maltrato físico o abuso sexual durante el embarazo y hasta un año después del parto. No obstante, Decker et al. (2004) han identificado que el embarazo puede constituir un factor protector para algunas de las mujeres que permanecen con su pareja; sin embargo, un quinto de las mujeres experimentan un incremento del número de factores de riesgo de letalidad una vez embarazadas.

Una de las expresiones de violencia más características en casos de homicidio a la mujer en el contexto doméstico, lo constituye la utilización de estrangulamiento o asfixia, ya sea como medio comisivo del delito o como forma de violencia anexa (Campbell et al., 2003; Dobash et al., 2007).

Por su parte, diversos autores han recalcado la relevancia de la utilización de armas como factor de riesgo de femicidio (Campbell et al., 2003; Dobash et Al., 2007; Echeburúa et al., 2009), lo cual resulta de elevado peligro no solo por el potencial letal asociado, sino también por la alienación y cosificación que implica la figura de la víctima para el agresor.

Junto con esto, el acoso por parte del agresor hacia la víctima ha sido estudiado por diversos autores, existiendo posturas divergentes. Por una parte, tanto Campbell et al. (2003) como Echeburúa et al. (2009) reconocen su aporte específico, dado que, de acuerdo a sus hallazgos, resulta más propio del homicidio hacia la mujer en la pareja que de la violencia doméstica. Concordantemente con lo anterior, el estudio de McFarlane et al. (2002) concluye que las conductas de acoso preceden al homicidio íntimo en el 68\% de los casos, particularmente a través de tres manifestaciones: seguir o espiar a la víctima, tratar de comunicarse con la víctima de modos que ella no deseaba y/o hacer llamadas no deseadas a la afectada. Por oposición, Soria (2005) señala que, si bien la conducta de acoso es común entre los agresores domésticos (45.7\%), no es posi- 
ble hacer distinciones entre el grupo de homicidas y maltratadores domésticos, respecto a la presencia de esta conducta, su causa o duración.

En relación con la agresión sexual, existe un nivel de consenso bastante elevado entre los investigadores, señalando que el sexo forzado con la víctima incrementa el riesgo de homicidio hacia la mujer en la relación de pareja (Echeburúa et al., 2009), aproximándose a la significación estadística (Campbell et al., 2003). Este tipo de violencia ha sido identificada por parte de Dobash et al. (2007) en este tipo de casos, pero no en violencia doméstica, reportando significativamente más factores de riesgo que aquellas que no han sufrido violencia de tipo sexual, sino solo física (McFarlane et al., 2005).

Adicionalmente, las amenazas del agresor contra la víctima o sus cercanos constituyen un importante factor de riesgo en este tipo de delitos (Campbell et al., 2003). A este respecto, McFarlane et al. (2002) encontraron correlación positiva con 7 formas de amenazas: amenazar a la víctima con un arma, herir mascotas a propósito, amenazar con herir a los niños si la víctima lo deja, amenazar de muerte a la víctima, amenazar a la familia de la víctima, dejar notas aterrorizantes y dejar mensajes amenazantes. Para Echeburúa et al. (2009), particularmente las amenazas previas con arma y amenazas de muerte incrementan la probabilidad de conducta letal. Según este autor, las amenazas y la violencia psicológica desplegada podrían ser comprendidas también a partir del comportamiento del agresor cruel y despectivo hacia la víctima.

Cabe señalar que, con frecuencia, la conducta violenta del autor está relacionada con celos irracionales y violentos (Campbell et al., 2003; Echeburúa et al. 2009), lo que genera el despliegue de una conducta controladora hacia la víctima. Esto, en conjunto con la interrupción de la convivencia, incrementa sustancialmente el riesgo de letalidad en la relación, efecto que es menor cuando esta variable se presenta por separado (Campbell et al., 2003).

Es necesario tener presente que la conducta maltratadora se asienta en un sistema de creencias que lo valida y permite, siendo un factor predictivo la justificación del agresor respecto a la violencia desplegada. Al respecto, Dobash y Dobash (2011, p. 130) analizan las cogniciones de hombres encarcelados por el homicidio de sus parejas femeninas, concluyendo que "los hombres utilizan la violencia para hacer cumplir normas rígidas sobre la base de sus creencias acerca de las relaciones de parejas (sic)". De este modo, la agresión es explicada ya sea desde su propio estado (estrés, alcohol o drogas), o bien desde la provocación de la víctima (Echeburúa et al., 2009). Concordantemente con lo anterior, Dobash y Dobash (2011) reconocen la racionalización frecuente de su comportamiento, así como la atribución de culpas a terceros (los padres, el sistema, la víctima), dada su incapacidad de aceptar la propia responsabilidad, sentir remordimiento por sus actos y empatizar con la víctima: "A partir de sus creencias sobre los comportamientos adecuados para las mujeres y los hombres en las relaciones íntimas, justifica la respuesta violenta, producto de los defectos de la mujer, como compañera íntima, madre y/o dueña de casa" (p. 130).

Finalmente, se reitera un principio básico de la psicología, el cual señala que el mejor predictor de la conducta futura es la conducta pasada (Ajzen, 1991). Desde esta perspectiva, los intentos de homicidio pasados constituyen un relevante factor de riesgo para que un acto de esta naturaleza se materialice en el futuro, principio que ha sido avalado por investigaciones como las de Campbell et al. (2003).

\section{Factores de riesgo asociados al contexto}

Frente a problemáticas de violencia doméstica, los Estados y la sociedad civil realizan esfuerzos por minimizar el riesgo de homicidio de la mujer en la relación de pareja, implementando políticas públicas destinadas a promover la denuncia de este tipo de casos, instalando casas de acogida orientadas a resguardar su integridad, programas de representación jurídica para asegurar la defensa de sus derechos e intereses, entre otros. La pregunta que cabe hacer, entonces, es de qué modo dichos recursos públicos y privados reducen o no el riesgo asociado en un tema tan crítico como este.

A este respecto, el estudio de Dugan et al. (2003) indica que diversos recursos de prevención están 
relacionados con menores niveles de homicidio al interior de la pareja, lo cual es comprendido en términos de su efectividad para reducir la exposición de la víctima a la violencia. Sin embargo, otros se asocian con un incremento en los niveles de homicidio, interpretado como un efecto de represalia o retaliación, en el cual la intervención gatilla la agresión, cuando no existe una adecuada reducción de la exposición.

Respecto a la actuación de las instituciones, un tema bastante controvertido dice relación con la denuncia de hechos constitutivos de violencia doméstica y la intervención policial. Sobre el particular, Campbell y sus colaboradores (2003) concluyen que un arresto previo por VIF reduce el riesgo de homicidio a la mujer en la pareja, sin embargo, la evasión de una detención o el incumplimiento de órdenes de alejamiento incrementa el riesgo asociado.

En relación con el acceso a armas de fuego, Richardson y Mercy (2006) concluyen que, comparativamente, el número de asesinatos íntimos se reduce en un 7\% en aquellos estados de E.E. U.U. que incorporaron leyes de restricción de acceso a armas de fuego.

\section{Discusión y conclusiones}

A partir de este análisis sistemático de la literatura, es posible apreciar el cuerpo de investigaciones que se ha generado, con miras a identificar factores de riesgo que inciden en la probabilidad de homicidio de la mujer por parte de su pareja masculina. A continuación, se procede a concluir respecto a la evidencia disponible, haciendo presente las controversias que se manifiestan en la literatura en relación con varios de los factores de riesgo estudiados.

Respecto a las variables propias del ofensor, las investigaciones han evidenciado la relevancia de aquellos factores estresores, vinculados a una situación de precarización. Entre ellos se mencionan la inmigración (Echeburúa et al., 2009) y el desempleo del agresor (Campbell et al., 2003; Dobash et al., 2007).

Respecto al consumo de sustancias, no existe acuerdo entre los investigadores en el papel del alcohol antes o durante el hecho, existiendo autores que relevan su papel (Echeburúa et al., 2009; Sharps et al., 2001) y otros que lo descartan (Campbell et al., 2003, Dobash et al., 2004; Dobash et al., 2007). Tal como señalan Campbell et al. (2007), dicha relación no ha podido ser establecida con nitidez.

Un aspecto que concita acuerdo entre los investigadores es el historial de violencia y criminalidad del autor, tanto hacia parejas anteriores (Dobash et al., 2004; Dobash et al., 2007; Echeburúa et al., 2009) como hacia personas de la comunidad (Echeburúa et al., 2009). Estas manifestaciones de violencia pueden correlacionarse con la tenencia de armas, particularmente de fuego (Campbell et al., 2003; Campbell, Glass, Sharps, Laughon \& Bloom, 2007), puesto que como señalan Campbell et al. (2007) en EE. UU. cerca del 70\% de estos delitos se producen con armas de fuego.

Desde la perspectiva de salud mental, los autores han destacado la presencia de psicopatología (Dixon et al., 2008; Echeburúa et al., 2009; Liem et al., 2009) y suicidalidad (Campbell et al., 2003; Liem et al., 2009) como factores de riesgo. Al respecto, para Echeburúa y De Corral (2009), el suicidio es más frecuente entre los agresores que presentan un mayor nivel de integración familiar y social, lo cual se vincula a un intento por evitar las consecuencias negativas de los hechos (rechazo social y castigo penal).

En relación con los factores propios de la víctima, al igual que en el caso del autor, los autores relevan variables de vulnerabilidad social y exclusión, tales como la pertenencia a minorías étnicas (Puzone et al., 2000) o bien situación de dependencia, enfermedad o aislamiento (Echeburúa et al., 2009). Adicionalmente, otros estudios han hecho alusión a la inmigración como factor de riesgo (Echeburúa $\&$ De Corral, 2009). Por otra parte, la existencia de un hijo no biológico del autor incrementa el riesgo asociado (Campbell, 2003).

Ahora bien, la percepción del riesgo vital por la mujer, no presenta una asociación lineal con el riesgo existente (Campbell et al., 2003; Campbell, Webster \& Glass, 2009; Echeburúa et al., 2009), asociación que sí se manifiesta de modo más directo respecto de la retractación (Echeburúa et al., 2009). 
En relación con las variables propias de la pareja, la literatura arriba a conclusiones disímiles respecto a la diferencia de edad entre víctima y autor (Dobash et al., 2007; Shackelford \& Mouzos, 2005), así como al vínculo marital o de convivencia (Puzone et al., 2000; Shackelford \& Mouzos, 2005). Más allá del vínculo civil, la cohabitación ha sido extensamente estudiada, no existiendo acuerdo de los investigadores respecto a su incidencia en el resultado de homicidio (Campbell et al., 2003; Dobash et al., 2007). Sin embargo, es claro que el término de la relación o la amenaza de aquello se asocia a riesgo elevado (Campbell et al., 2003; Dobash et al., 2004; Liem et al., 2009). A este respecto, Campbell et al. (2007) añaden que el alejamiento puede producirse en la forma de separación física y/o legal, siendo la conjunción de ambas la que acarrea mayor riesgo letal.

Por otra parte, se suele presentar un extenso historial de violencia hacia la víctima, lo cual puede incluir violencia física grave, estrangulamiento, uso de armas, intentos de homicidio previos, conductas de acoso, agresiones sexuales, amenazas, celos violentos y conducta controladora por parte del autor (Campbell et al., 2003; Dobash et al., 2007; Dobash \& Dobash, 2011; Echeburúa et al., 2009; McFarlane et al., 2002; McFarlane et al., 2005). Más controvertidas resultan las manifestaciones de maltrato durante el embarazo (Decker et al., 2004; Taylor \& Nabors, 2009) y de acoso (Soria, 2005). En general, estas manifestaciones de violencia son justificadas y/o racionalizadas por parte del autor (Dobash \& Dobash, 2011; Echeburúa, 2009).

En este sentido, de acuerdo a conceptualizaciones teóricas como las que se derivan del Ciclo de la Violencia (Walker, 2009), se tiende a interpretar el homicidio doméstico como el punto culmine de una relación crónica de maltrato en escalada. Si bien es cierto que "la mayoría de los asesinatos al interior de la pareja fueron precedidos por violencia de pareja" (Campbell et al., 2007, p. 247), hay que tener presente que un porcentaje de ellos no manifiesta precursores.

Entre los factores de riesgo asociados al contexto, los autores señalan que numerosos recursos de prevención se correlacionan con menor riesgo de homicidio de la mujer en la pareja, sin embargo, otros se comportan de modo opuesto, dependiendo de su capacidad para reducir la exposición, así como de evitar el efecto de retaliación (Dugan et al., 2003). No obstante lo anterior, De Leon-Granados y Wells (2003) relativizan dichas conclusiones, al objetar la validez y confiabilidad de los indicadores agregados que se utilizan para medir los recursos dispuestos para la prevención de la violencia doméstica.

$\mathrm{Al}$ respecto, un aspecto particularmente sensible es la denuncia de violencia intrafamiliar y la actuación policial; en tanto las detenciones pueden reducir el riesgo o incrementarlo en caso de evasión de la detención o incumplimiento de las órdenes de alejamiento (Campbell et al., 2003). Sobre lo anterior, un estudio Loseke y Rauma (1983 en Andrés Pueyo, 2009) detecta que las intervenciones policiales y particularmente las órdenes de detención, incrementan el riesgo de maltrato grave como consecuencia. En palabras de Echeburúa y De Corral (2009, p. 140) “(...) una denuncia, si no hay protección efectiva de la mujer y no se cuenta con cierto apoyo social y familiar, puede ser una mala decisión".

Tal como es posible deducir de los párrafos precedentes, frente al riesgo que implica el asesinato de la mujer en la relación de pareja, la sociedad realiza esfuerzos por minimizar su probabilidad de ocurrencia, poniendo a disposición recursos de prevención, mecanismos de denuncia, intervención policial, así como la elaboración de leyes y políticas públicas específicas en la materia. Todo ello, con resultados a lo menos discutibles respecto al control del riesgo experimentado.

Esto, por cuanto se tiende a suponer que las respuestas a este fenómeno pasan fundamentalmente por el control, lo que lleva a promover y privilegiar la denuncia, la judicialización y acciones coercitivas por parte del Estado. Es necesario problematizar, por tanto, respecto a la enajenación del conflicto que se genera desde el aparato punitivo, más aún cuando la mujer no ha tomado la decisión de iniciar o mantener este camino, lo cual frecuentemente se asocia con retractaciones, que generan un gasto importante para el Estado 
y situaciones que incluso pueden acarrear riesgo vital para la afectada. Junto con esto, somos testigos de intervenciones psicosociales de corto plazo para las víctimas, que no consiguen modificar eficazmente las condiciones de vida y los lazos que perpetúan este ciclo de violencia. Esto advierte respecto al efecto de retaliación y a la posibilidad de intervenciones iatrogénicas por parte de las instituciones, en caso de carecer de sistema de protección que permita acompañar procesos tan complejos como los que se elicitan a consecuencia de la violencia doméstica.

De la misma manera, la preocupación por la salud mental de los autores es leída como un privilegio inmerecido, del cual el Estado y la sociedad civil no deben hacerse cargo, obviando así uno de los factores fundamentales de riesgo, no solo para la mujer vulnerada, sino también para otras en el futuro.

Frente a este escenario, el Estado y la sociedad civil deben generar condiciones que garanticen la seguridad de las afectadas tras la develación de la violencia, en condiciones de dignidad y sustentabilidad, contemplando tanto las necesidades de las mujeres como las de sus hijos, testigos y víctimas colaterales de esta forma de violencia.

Por otra parte, cabe señalar que todas las investigaciones identificadas dicen relación con la realidad de países como EE. UU., España, Reino Unido, Países Bajos y Australia. Llama la atención la carencia de estudios propios de la realidad latinoamericana, que permitan dar luces respecto a los factores de riesgo propios de nuestro contexto, permitiendo de este modo orientar las decisiones interventivas y de política pública local, que obedezcan a la particular idiosincrasia latinoamericana.

\section{Referencias}

Ajzen, I. (1991). The theory of planned behavior. Organizational Behavior And Human Decision Processes, 50(2), 179-211.

Andrés Pueyo, A. (2009). La predicción de la violencia contra la pareja. En E. Echeburúa, J. FernándezMontalvo \& P. De Corral (Eds.), Predicción del riesgo de homicidio y violencia grave en la relación depareja (pp. 21-55). Valencia: Centro Reina Sofía.
Campbell, J., Glass, N., Sharps, P., Laughon, K., \& Bloom, T. (2007). Intimate partner homicide. Review and implications of research and policy. Trauma, Violence and Abuse, 8(3), 246-269.

Campbell, J., Webster, D., \& Glass, N. (2009). The danger assessment: Validation of a lethality risk assessment instrument for intimate partner femicide. Journal of Interpersonal Violence, 24(4), 653-674.

Campbell, J., Webster, D., Koziol-McLain, J., Block, C., Campbell, D., Curry, M. A., et al. (2003). Risk factors for femicide in abusive relationships: Results from a multisite case control study. American Journal of Public Health, 93(7), 1089-1097.

Chile, Programa de las Naciones Unidas para el Desarro1lo (2004, septiembre/octubre). Estudio ONU propone medidas para combatir asesinato de mujeres (Boletín Informativo del Programa de las Naciones Unidas para el Desarrollo, № 5). Recuperado de http://www.pnud.cl/boletin/enero2005/cont07.htm

Decker, M., Martin, S., \& Moracco, K. (2004). Homicide risk factors among pregnant women abused by their partners: Who leaves the perpetrator and who stays? Violence Against Women, 10(5), 498-513.

De Leon-Granados, W., \& Wells, W. (2003). The reliability and validity of measures of domestic violence resources as used in intimate partner homicide research. Violence Against Women, 9(2), 148-162.

Dixon, L., Hamilton-Giachritsis, C., \& Browne, K. (2008). Classifying partner femicide. Journal of Interpersonal Violence, 23(1), 74-93.

Dobash, R., \& Dobash, R. (2011). What were they thinking? Men who murder an intimate partner. Violence Against Woman, 1(1), 113-134.

Dobash, R., Dobash, R., Cavanagh, K., \& Lewis, R. (2004). Not an ordinary killer - just an ordinary guy. Violence Against Woman, 10(6), 557-605.

Dobash, R., Dobash, R., Cavanagh, K., \& Medina-Ariza, J. (2007). Lethal and nonlethal violence against an intimate female partner: Comparing male murders to nonlethal abusers. Violence Against Women, 13(4), 329-353.

Dugan, L., Rosenfield, R., \& Nagin, D. (2003). Exposure reduction or retaliation? The effects of domestic violence resources on intimate-partner homicide. Law EO Society Review, 37(1), 169-198. 
Echeburúa, E., \& De Corral, P. (2009). El homicidio en la relación de pareja: un análisis psicológico. Eguzkilore, 23, 139-150.

Echeburúa, E., \& Fernández-Montalvo, J. (2007). Male batterers with and without psychopaty. Internacional Journal of Offender Therapy and Comparative Criminology, 51(3), 254-263.

Echeburúa, E., Fernández-Montalvo, J., De Corral, P., \& López-Goñi, J. (2009). Assessing risk markers in intimate partner femicide and severe violence: $\mathrm{A}$ new assessment instrument. Journal of Interpersonal Violence, 24(6), 925-939.

Heise, L., Ellsberg, M., \& Gottemoeller, M. (1999). Ending violence against women (Population Reports, Serie L, № 11). Baltimore, MD: Johns Hopkins University School of Public Health.

Instituto Interamericano de Derechos Humanos \& Consejo Centroamericano de Procuradores de Derechos Humanos (2006, agosto). Situación y análisis del femicidio en la Región Centroamericana (Informe Regional). Ciudad de Guatemala: Autores. Disponible en http://www.cawn.org/espanol/ publicaciones/documentos/otras/Femicidio\%20 oficial.pdf

Krug, E., Dahlberg, L., \& Mercy, J. (2003). Informe mundial sobre la violencia y la salud. Washington: OPS.

Liem, M., Hengeveld, M., \& Koenraadt, F. (2009). Domestic homicide followed by parasuicide: A comparison with homicide and parasuicide. International Journal of Offender Therapy and Comparative Criminology, 53(5), 497-516.

McFarlane, J., Malecha, A., Jist, J., Watson, K., Batten, E., Hall, I., \& Smith, S. (2005). Intimate partner sexual assault against women and associated victim substance use, suicidality, and risk factors for femicide. Issues in Mental Health Nursing, 26(9), 953-967.

McFarlane, J., Campbell, J., \& Watson, K. (2002). Intimate partner stalking and femicide: Urgent impli- cations for women's safety. Behavioral Sciences and Law, 20(1-2), 51-68.

Organización Mundial de la Salud (2005). Estudio multipaís de la OMS sobre salud de la mujer y violencia doméstica contra la mujer. Ginebra: Autor.

Puzone, C., Saltzman, E., Kresnow, M., Thompson, M., \& Mercy, J. (2000). National trends in intimate partner homicide. Violence Against Women, 6(4), 409-426.

Richardson, E., \& Mercy, J. (2006). Do Laws restricting access to firearms by domestic violence offenders prevent intimate partner homicide? Evaluation Review, 30(3), 313-346.

Russell, D., \& Harmes, R. (2006). Feminicidio: una perspectiva global. México: Universidad Nacional Autónoma de México.

Sanmartín, J., Iborra I., García, I., \& Martínez, P. (2010). III Informe Internacional Violencia Contra la Mujer en las Relaciones de Pareja. Estadísticas y legislación. Valencia: Centro Reina Sofía para el Estudio de la Violencia.

Shackelford, T., \& Mouzos, J. (2005). Partner killing by men in cohabiting and marital relationships. A comparative, cross-national analysis of data from Australia and the United States. Journal of Interpersonal Violence, 20(10), 1310-1324.

Sharps, P., Campbell, J., Campbell, D., Gary, F., \& Webster, D. (2001). The role of alcohol use in intimate partner femicide. The American Journal on Addictions, 10(2), 122-135.

Soria, M. A. (2005). La conducta de acoso en maltratadores y homicidas domésticos. Intervención Psicosocial, 14(2), 177-189.

Taylor, R., \& Nabors, E. (2009). Pink or blue... black and blue? Examining pregnancy as a predictor of intimate partner violence and femicide. Violence Against Women, 15(11), 1273-1293.

Walker, L. (2009). The battered woman syndrome. New York: Springer Publishing Company. 